Article

\title{
The Extracts of Artemisia absinthium L. Suppress the Growth of Hepatocellular Carcinoma Cells through Induction of Apoptosis via Endoplasmic Reticulum Stress and Mitochondrial-Dependent Pathway
}

\author{
Xianxian Wei ${ }^{\dagger}$, Lijie Xia ${ }^{\dagger}$, Dilinigeer Ziyayiding, Qiuyan Chen, Runqing Liu, Xiaoyu Xu and \\ Jinyao Li $*$ (1)
}

Xinjiang Key Laboratory of Biological Resources and Genetic Engineering, College of Life Science and Technology, Xinjiang University, Urumqi, Xinjiang 830046, China; 15099141611@189.cn (X.W.); xialijie1219@163.com (L.X.); dilnigar9696@sina.com (D.Z.); m15276567620_1@163.com (Q.C.); Lucyducy@163.com (R.L.); 15276654427@139.com (X.X.)

* Correspondence: ljyxju@xju.edu.cn; Tel.: +86-991-8583259; Fax: +86-991-8583517

+ These two authors contributed equally.

Academic Editor: Roberto Fabiani

Received: 27 January 2019; Accepted: 28 February 2019; Published: 5 March 2019

\begin{abstract}
Artemisia absinthium L. has pharmaceutical and medicinal effects such as antimicrobial, antiparasitic, hepatoprotective, and antioxidant activities. Here, we prepared A. absinthium ethanol extract (AAEE) and its subfractions including petroleum ether (AAEE-Pe) and ethyl acetate (AAEE-Ea) and investigated their antitumor effect on human hepatoma BEL-7404 cells and mouse hepatoma H22 cells. The cell viability of hepatoma cells was measured by 3-(4,5-dimethylthiazol-2-yl)-2,5diphenyltetrazolium bromide (MTT) assay. The apoptosis, cell cycle, mitochondrial membrane potential $(\Delta \psi \mathrm{m})$, and reactive oxygen species (ROS) were analyzed by flow cytometry. The levels of proteins in the cell cycle and apoptotic pathways were detected by Western blot. AAEE, AAEE-Pe, and AAEE-Ea exhibited potent cytotoxicity for both BEL-7404 cells and $\mathrm{H} 22$ cells through the induction of cell apoptosis and cell cycle arrest. Moreover, AAEE, AAEE-Pe, and AAEE-Ea significantly reduced $\Delta \psi \mathrm{m}$, increased the release of cytochrome $\mathrm{c}$, and promoted the cleavage of caspase-3, caspase- 9 , and poly(ADP-ribose) polymerase (PARP) in BEL-7404 and H22 cells. AAEE, AAEE-Pe, and AAEE-Ea significantly upregulated the levels of ROS and C/EBP-homologous protein (CHOP). Further, AAEE, AAEE-Pe, and AAEE-Ea significantly inhibited tumor growth in the $\mathrm{H} 22$ tumor mouse model and improved the survival of tumor mice without side effects. These results suggest that AAEE, AAEE-Pe, and AAEE-Ea inhibited the growth of hepatoma cells through induction of apoptosis, which might be mediated by the endoplasmic reticulum stress and mitochondrial-dependent pathway.
\end{abstract}

Keywords: Artemisia absinthium; apoptosis; endoplasmic reticulum stress; mitochondrial-dependent pathway

\section{Introduction}

Hepatocellular carcinoma (HCC) is one of the most common malignant tumors and was the sixth most commonly diagnosed cancer and the fourth leading cause of cancer death worldwide in 2018, with about 841,000 new cases and 782,000 deaths annually [1,2]. The main risk factors for HCC are chronic infection with hepatitis B virus (HBV) or hepatitis C virus (HCV), aflatoxin-contaminated foodstuffs, heavy alcohol intake, obesity, smoking, and type 2 diabetes [3]. Currently, therapeutic options for the treatment of HCC include liver resection, transplantation, palliative intra-arterial therapies, immunotherapy strategies, and so on [4,5]. However, the prognosis of most patients with 
HCC is poor [6]. For HCC treatment, the main drugs, including oxaliplatin and sorafenib, remain unsatisfactory because of their side effects and multidrug resistance $[7,8]$. Therefore, it is urgent to develop novel therapeutic agents to treat HCC.

Artemisia absinthium L. belongs to the Asteraceae family and is commonly known as wormwood. The chemical components of A. absinthium include sesquiterpene lactone, sesquiterpene lactone-pinene, $\beta$-thujone, $\alpha$-thujone, sabinyl acetate, and $\beta$-thujone [9]. A. absinthium has pharmaceutical and medicinal effects such as antimicrobial [9], insecticidal [10], antiparasitic [11], antitumor [12], antipyretic [13], hepatoprotective [14,15], and antioxidant activities [16,17]. In the present study, A. absinthium ethanol extract (AAEE) and its subfractions including petroleum ether (AAEE-Pe) and ethyl acetate (AAEE-Ea) were prepared, and their antitumor effects on HCC were investigated both in vitro and in vivo. AAEE, AAEE-Pe, and AAEE-Ea selectively inhibited the growth of hepatoma cells both in vitro and in vivo without cytotoxic effects on normal hepatic cells. Moreover, these extracts could arrest the cell cycle at the G2/M phase and induce apoptosis through endoplasmic reticulum (ER) stress and the mitochondrial-dependent pathway in human hepatoma BEL-7404 cells and mouse hepatoma H22 cells, and they might be used as safe and effective agents for the treatment of HCC.

\section{Results}

\subsection{AAEE, AAEE-Pe, and AAEE-Ea Suppress the Growth of BEL-7404 and H22 Cells In Vitro}

To investigate the anti-proliferative effects of AAEE, AAEE-Pe, and AAEE-Ea, BEL-7404 and $\mathrm{H} 22$ cells were treated with 25, 75, and $150 \mu \mathrm{g} / \mathrm{mL}$ of AAEE, AAEE-Pe, and AAEE-Ea. After $24 \mathrm{~h}$, the morphology of BEL-7404 and H22 cells was observed with an inverted microscope. Compared to untreated cells, BEL-7404 and H22 cells treated with AAEE, AAEE-Pe, and AAEE-Ea became shrunk and round, and cell numbers were reduced in a dose-dependent manner (Figure 1A). Cell viability was detected by MTT assay after treatment for 24, 48, and 72 h. The viability of BEL-7404 and H22 cells was dose- and time-dependently decreased after treatment with AAEE, AAEE-Pe, or AAEE-Ea (Figure 1B). The $\mathrm{IC}_{50}$ (50\% inhibitory concentration) values of AAEE, AAEE-Pe, and AAEE-Ea for BEL-7404 and $\mathrm{H} 22$ cells at 24, 48, and $72 \mathrm{~h}$ are shown in Table 1 . The $\mathrm{IC}_{50}$ values of $\mathrm{H} 22$ cells followed the order AAEE-Pe $\leq$ AAEE $<$ AAEE-Ea. The $\mathrm{IC}_{50}$ values of BEL-7404 cells followed the order AAEE $\leq$ AAEE-Pe < AAEE-Ea. The effect of AAEE, AAEE-Pe, and AAEE-Ea was also detected on normal liver cells NCTC1469. AAEE and AAEE-Pe showed some cytotoxicity on NCTC1469 cells, but it was much lower than that of BEL-7404 and H22 cells. AAEE-Ea has no cytotoxicity on NCTC1469 cells (Figure 1C). These results suggest that AAEE, AAEE-Pe, and AAEE-Ea selectively inhibited the growth of hepatoma cells in vitro.

Table 1. $\mathrm{IC}_{50}$ values of AAEE, AAEE-Pe, and AAEE-Ea for BEL-7404 and H22 cells.

\begin{tabular}{ccccc}
\hline & & \multicolumn{3}{c}{ IC $_{\mathbf{5 0}}$} \\
\cline { 3 - 5 } & & $\mathbf{2 4} \mathbf{~ h}$ & $\mathbf{4 8} \mathbf{~ h}$ & $\mathbf{7 2 ~} \mathbf{~}$ \\
\hline \multirow{3}{*}{ H22 cells } & AAEE & 59.16 & 33.40 & 14.71 \\
& AAEE-Pe & 32.91 & 29.64 & 23.38 \\
& AAEE-Ea & 97.76 & 43.57 & 25.69 \\
\hline \multirow{3}{*}{ BEL-7404 cells } & AAEE & 89.86 & 56.86 & 60.39 \\
& AAEE-Pe & 98.71 & 75.88 & 89.51 \\
& AAEE-Ea & 171.7 & 82.04 & 71.97 \\
\hline
\end{tabular}




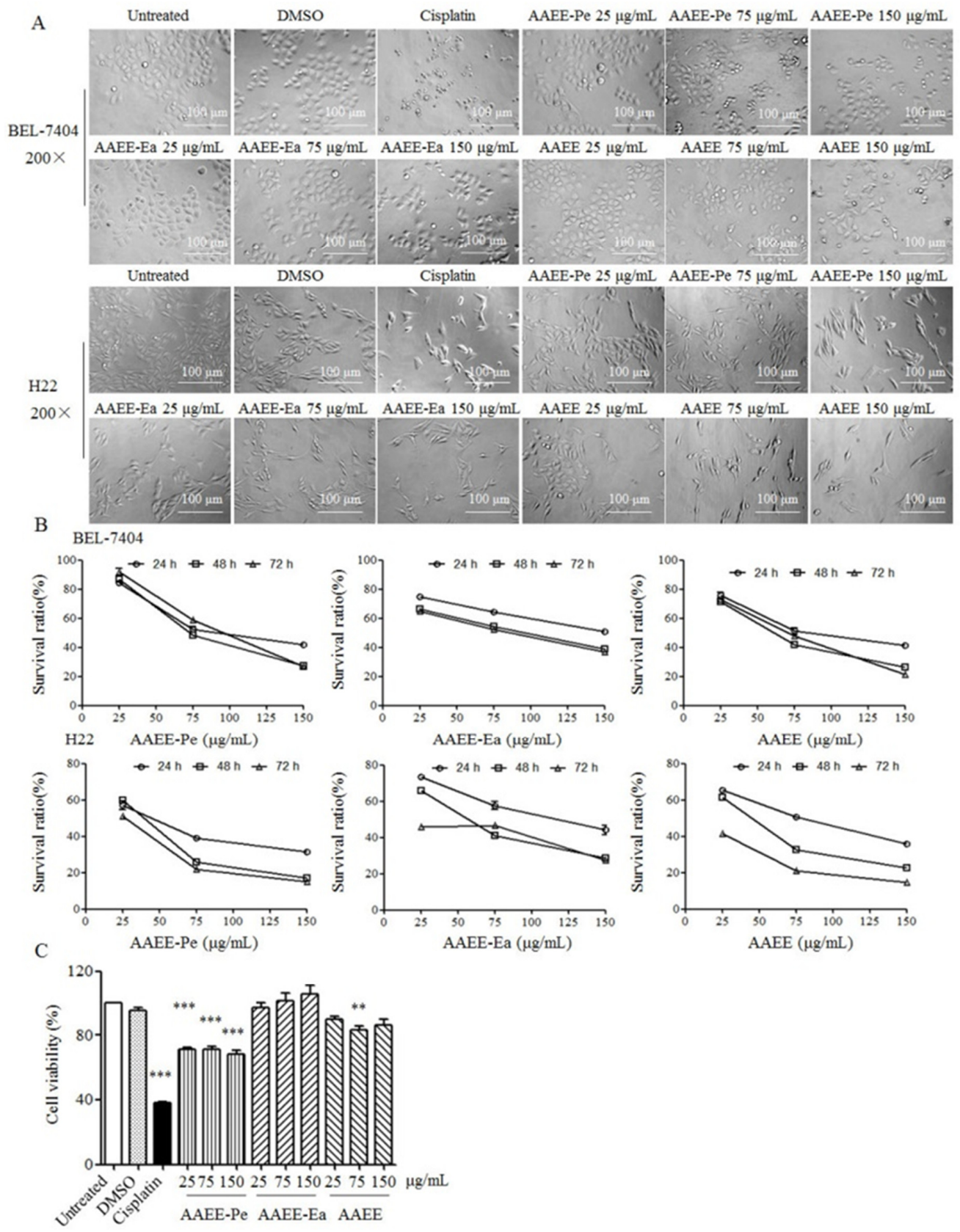

Figure 1. The effect of $A$. absinthium ethanol extract (AAEE) and its petroleum ether (AAEE-Pe) and ethyl acetate (AAEE-Ea) subfractions on the growth of BEL-7404, H22, and NCTC1469 cells. Cells were treated with different concentrations of AAEE, AAEE-Pe, and AAEE-Ea. (A) After $24 \mathrm{~h}$, the morphology of BEL-7404 and H22 cells was observed by inverted microscope. (B) After 24, 48, and $72 \mathrm{~h}$, the viability of BEL-7404 and H22 cells was detected by MTT assay. (C) After $24 \mathrm{~h}$, the viability of NCTC1469 cells was detected by MTT assay. ${ }^{* *} p<0.01,{ }^{* * *} p<0.001$ compared to Untreated.

\subsection{AAEE, AAEE-Pe, and AAEE-Ea Induce Apoptosis in BEL-7404 and H22 Cells}

To study whether AAEE, AAEE-Pe, and AAEE-Ea induce apoptosis, BEL-7404 and H22 cells were stained with Annexin V and propidium iodide (PI) after treatment and analyzed by flow cytometry. The frequencies of apoptotic BEL-7404 and B22 cells were significantly increased by each of AAEE, AAEE-Pe, and AAEE-Ea in a dose-dependent manner (Figure 2A,B). AAEE, AAEE-Pe, and AAEE-Ea did not induce the necrosis of BEL-7404 cells but significantly induced necrosis of H22 cells (Figure 2A,B). The pro- and anti-apoptotic members of the B-cell lymphoma-2 (BCL-2) protein family 
serve important roles in the regulation of cell apoptosis. After treatment with AAEE, AAEE-Pe, and AAEE-Ea, the levels of pro-apoptotic Bax and anti-apoptotic Bcl-2 in BEL-7404 and H22 cells were upregulated and downregulated, respectively. The ratios of $\mathrm{Bax} / \mathrm{Bcl} 2$ significantly increased upon AAEE, AAEE-Pe, and AAEE-Ea treatment (Figure 2C). The results indicate that AAEE, AAEE-Pe, and AAEE-Ea inhibited the growth of BEL-7404 and H22 cells through the induction of apoptosis.
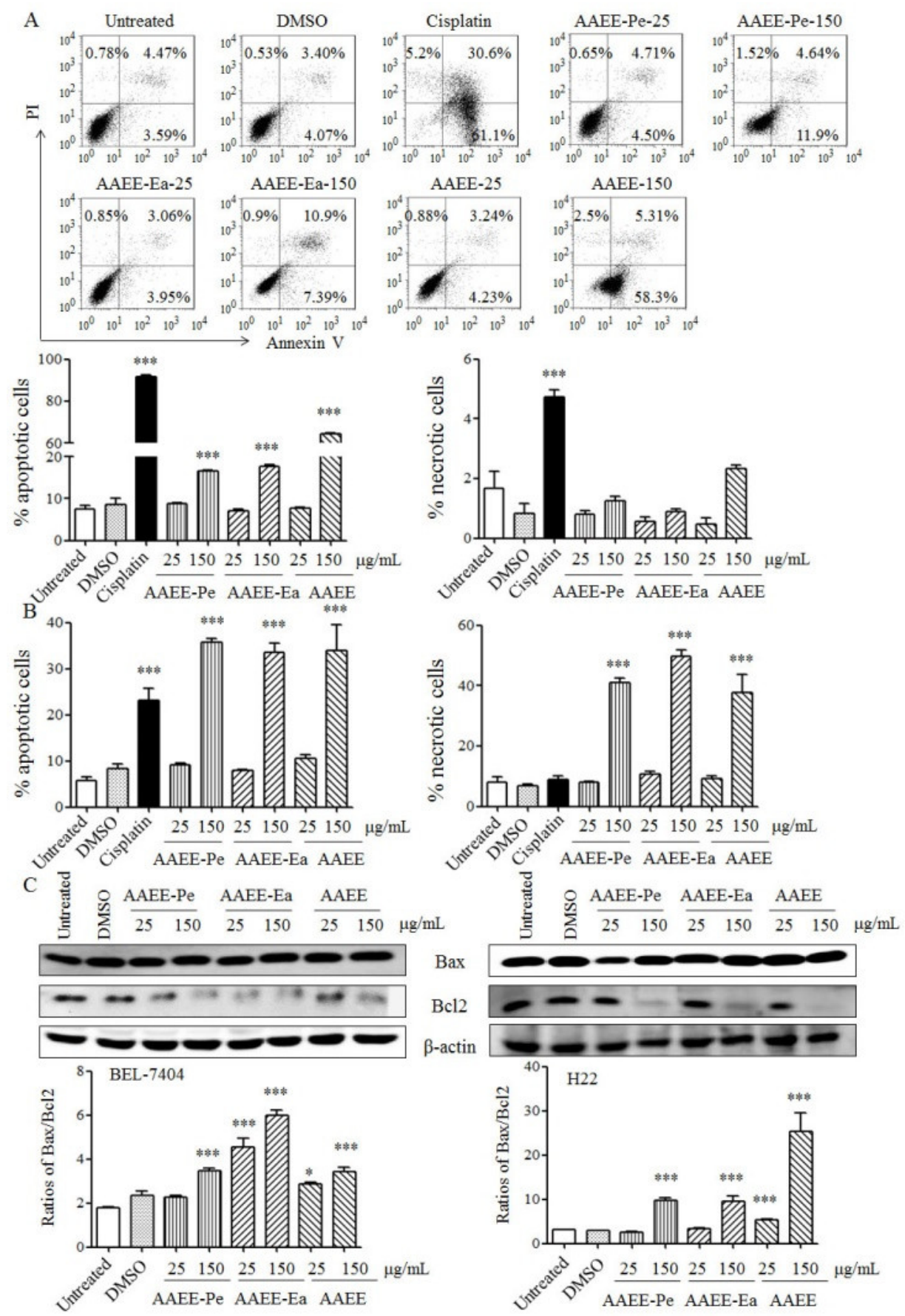

Figure 2. AAEE, AAEE-Pe, and AAEE-Ea induced apoptosis in BEL-7404 and H22 cells. Cells were treated with different concentrations of AAEE, AAEE-Pe, and AAEE-Ea for $24 \mathrm{~h}$. After staining with Annexin V and PI, BEL-7404 (A) and H22 (B) cells were analyzed by flow cytometry. (C) Proteins were isolated, and the levels of Bax and Bcl-2 were analyzed by Western blot. ${ }^{*} p<0.05 ;{ }^{* * *} p<0.001$ compared to Untreated. 


\subsection{AAEE, AAEE-Pe, and AAEE-Ea Induce Cell Cycle Arrest in BEL-7404 and H22 Cells}

The morphological characteristics of apoptosis include chromatin condensation and DNA fragmentation. After treatment with AAEE, AAEE-Pe, and AAEE-Ea, BEL-7404 and H22 cells were stained with Hoechst 33258 and observed by inverted fluorescence microscopy. The nuclei of untreated cells showed homogeneous staining, while the nuclei of cells treated with AAEE, AAEE-Pe, and AAEE-Ea showed condensed chromatin (Figure 3A), suggesting that AAEE, AAEE-Pe, and AAEE-Ea induce apoptosis in BEL-7404 and H22 cells.

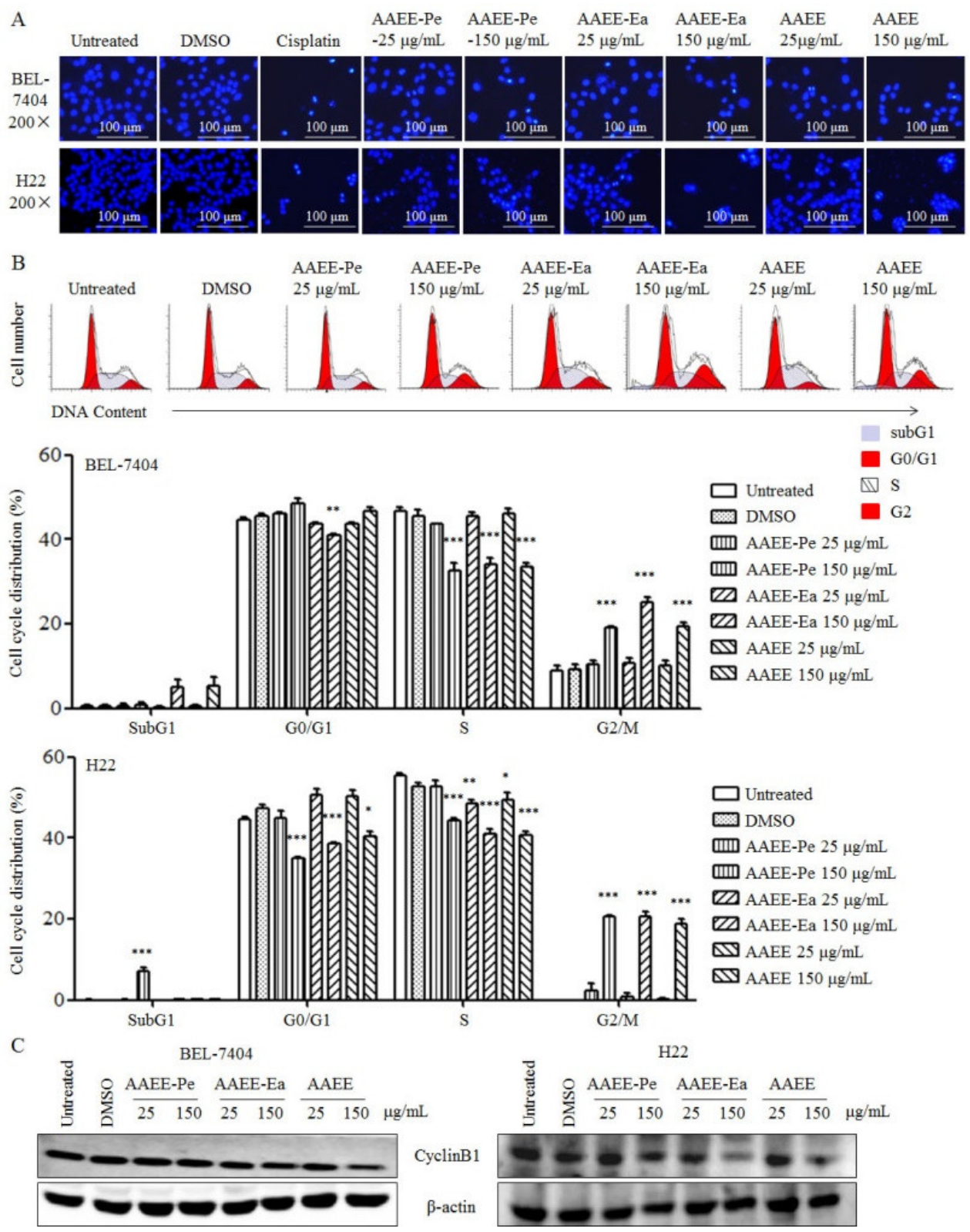

Figure 3. AAEE, AAEE-Pe, and AAEE-Ea induced cell cycle arrest in BEL-7404 and H22 cells. BEL-7404 and $\mathrm{H} 22$ cells were treated with different concentrations of AAEE, AAEE-Pe, and AAEE-Ea for $24 \mathrm{~h}$. (A) BEL-7404 and H22 cells were stained with Hoechst 33258 and observed by inverted fluorescence microscope. (B) DNA contents in BEL-7404 cells were analyzed by flow cytometry and are shown in the upper panels. The summaries of the cell cycle distributions in BEL-7404 and H22 cells are shown in the middle and bottom panels, respectively. (C) Expression of Cyclin B1 was analyzed by Western blot. ${ }^{*} p<0.05 ;{ }^{* *} p<0.01 ;{ }^{* * *} p<0.001$ compared to Untreated. 
Next, the distribution of the cell cycle in BEL-7404 and H22 cells was detected by PI staining after treatment with AAEE, AAEE-Pe, and AAEE-Ea for $24 \mathrm{~h}$. We observed that cells in the G2/M phase were significantly increased and cells in the $S$ phases were significantly decreased upon AAEE, AAEE-Pe, and AAEE-Ea treatment (Figure 3B), indicating that AAEE, AAEE-Pe, and AAEE-Ea arrested the cell cycle of BEL-7404 and H22 cells at the G2/M phase. Consistently, AAEE, AAEE-Pe, and AAEE-Ea reduced the expression of cyclin B1 in BEL-7404 and H22 cells (Figure 3C). The results suggest that AAEE, AAEE-Pe, and AAEE-Ea induced apoptosis and cell cycle arrest in hepatoma cells.

\subsection{AAEE, AAEE-Pe, and AAEE-Ea Reduce Mitochondrial Membrane Potential ( $\triangle \psi m$ )}

Mitochondrial membrane integrity is strictly regulated by the pro- and anti-apoptotic members of the BCL-2 protein family. After treatment with AAEE, AAEE-Pe, and AAEE-Ea for $24 \mathrm{~h}$, the $\Delta \psi \mathrm{m}$ was measured by flow cytometry using JC-1 as a fluorescent dye. As shown in Figure 4A, AAEE, AAEE-Pe, and AAEE-Ea significantly reduced the $\Delta \psi \mathrm{m}$ values of BEL-7404 and H22 cells in a dose-dependent manner. Consistently, AAEE, AAEE-Pe, and AAEE-Ea promoted the release of cytochrome $c$ in the cytosol of BEL-7404 and H22 cells. The activation of caspases is generally considered to be a key hallmark of apoptosis. We also observed that the levels of cleaved caspase-3 and caspase-9 were increased upon AAEE, AAEE-Pe, and AAEE-Ea treatment, which promoted the cleavage of poly(ADP-ribose) polymerase (PARP) in both BEL-7404 and H22 cells (Figure 4B). The results indicate that AAEE, AAEE-Pe, and AAEE-Ea induced apoptosis in hepatoma cells through the mitochondria-dependent pathway.

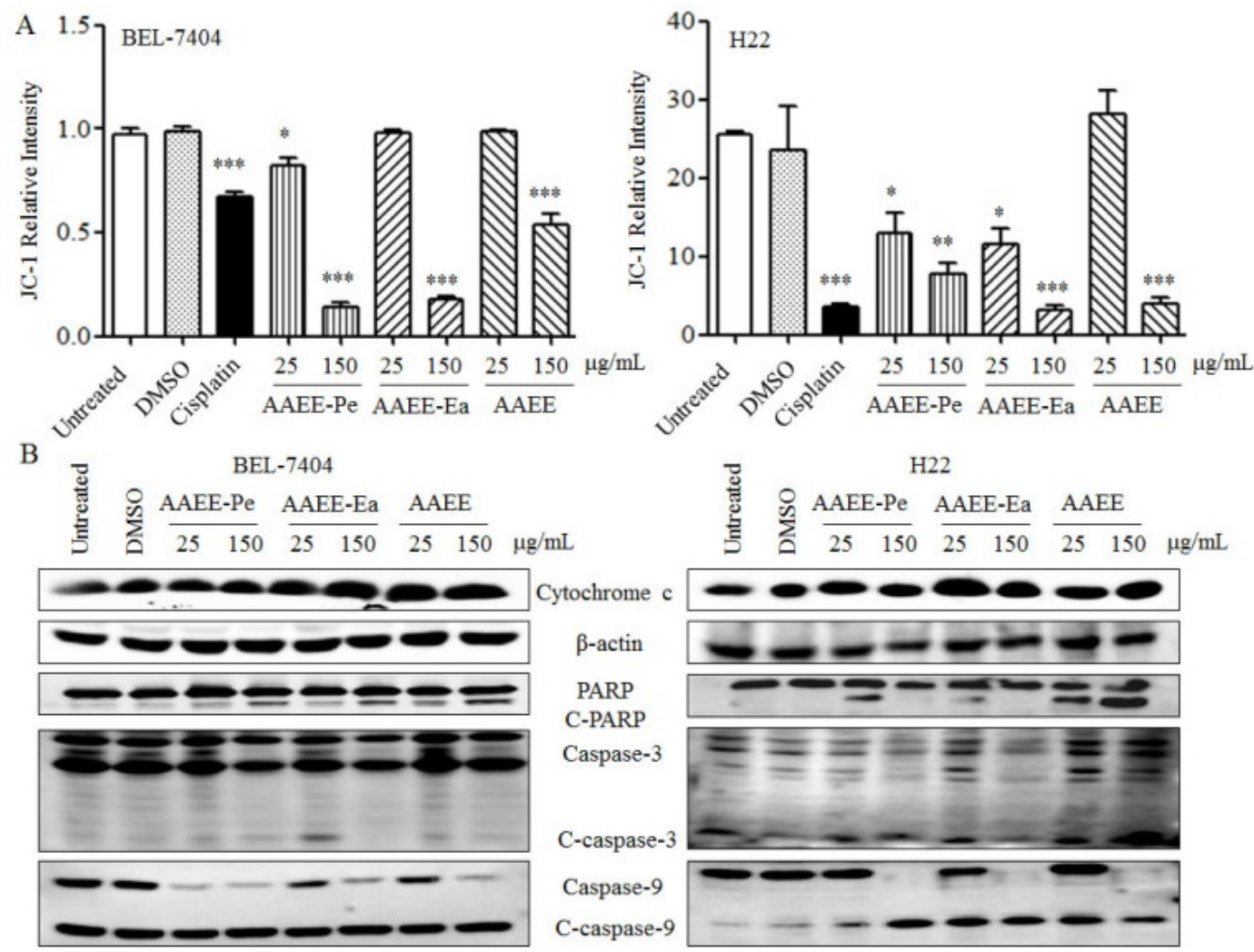

Figure 4. The mitochondria-dependent apoptosis induced by AAEE, AAEE-Pe, and AAEE-Ea. BEL-7404 and H22 cells were treated with different concentrations of AAEE, AAEE-Pe and AAEE-Ea for $24 \mathrm{~h}$. (A) Cells were stained with JC-1 dye and analyzed by flow cytometry. (B) Total protein was isolated to detect the release of cytochrome $c$ and the levels of poly(ADP-ribose) polymerase (PARP), cleaved-PARP (C-PARP), cleaved-caspase-3, caspase-3, cleaved-caspase- 9 , and caspase- 9 by Western blot. ${ }^{*} p<0.05 ;{ }^{* *} p<0.01 ;{ }^{* * *} p<0.001$ compared to Untreated. 


\subsection{AAEE, AAEE-Pe, and AAEE-Ea Promote Reactive Oxygen Species (ROS) Generation and ER Stress}

To examine the effects of AAEE, AAEE-Pe, and AAEE-Ea on oxidative stress, ROS generation was detected by flow cytometry at the indicated time points. After treatment with AAEE, AAEE-Pe, and AAEE-Ea, ROS levels were significantly increased at $6 \mathrm{~h}$, reached a peak at $12 \mathrm{~h}$, and then were maintained to $24 \mathrm{~h}$ in BEL-7404 cells (Figure 5A). Similarly, AAEE, AAEE-Pe, and AAEE-Ea dose-dependently increased ROS levels in H22 cells after treatment for $24 \mathrm{~h}$ (Figure 5B).
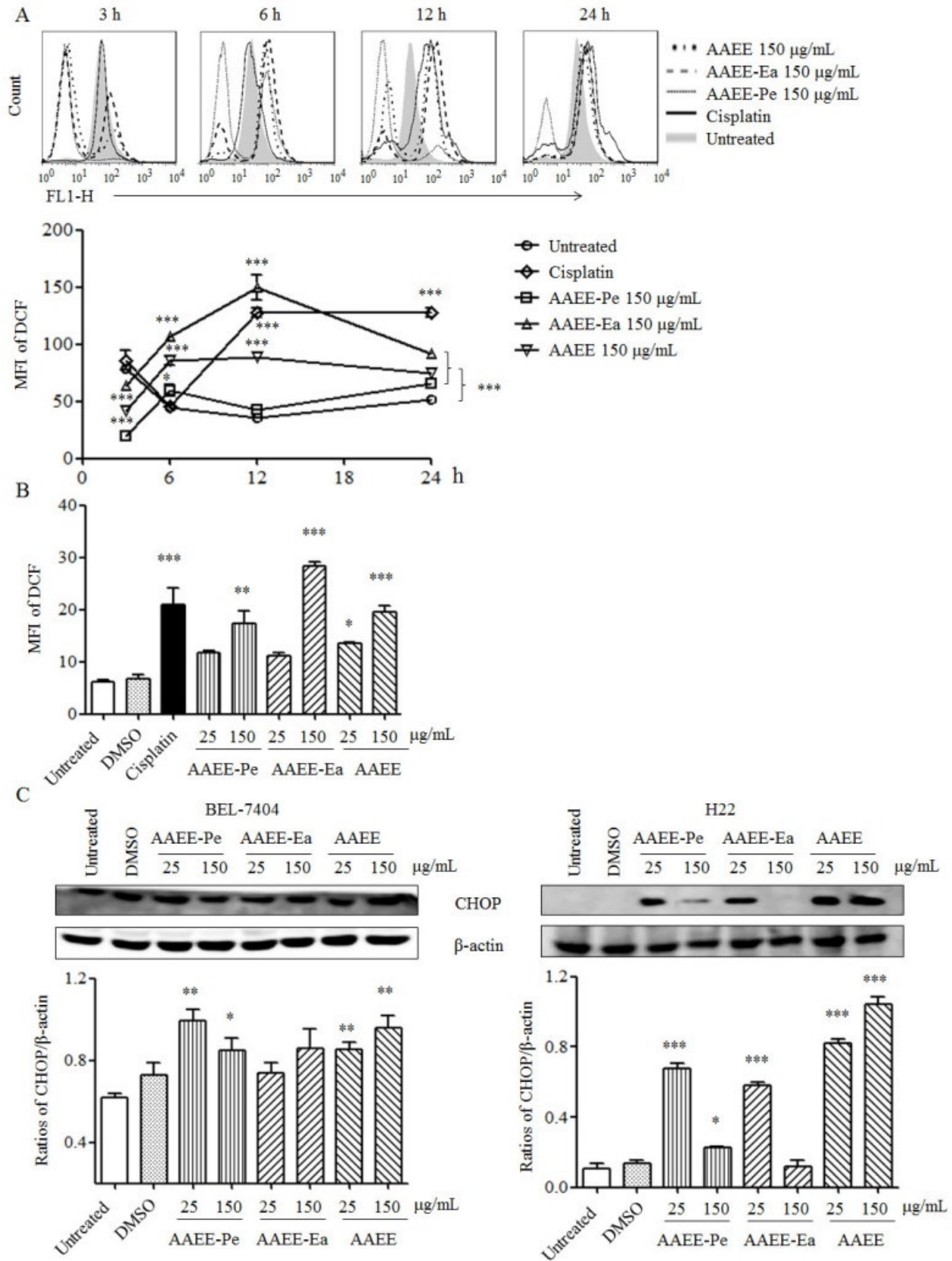

Figure 5. AAEE, AAEE-Pe, and AAEE-Ea induced ROS generation and ER stress. (A) BEL-7404 cells were treated with $150 \mu \mathrm{g} / \mathrm{mL}$ of AAEE, AAEE-Pe, and AAEE-Ea and the levels of ROS were analyzed by flow cytometry at indicated time points. (B) $\mathrm{H} 22$ cells were treated with different concentrations of AAEE, AAEE-Pe, and AAEE-Ea for $24 \mathrm{~h}$ and the levels of ROS were analyzed by flow cytometry. (C) BEL-7404 and H22 cells were treated with different concentrations of AAEE, AAEE-Pe, and AAEE-Ea for $24 \mathrm{~h}$. Cell lysates were used to analyze the levels of CHOP by Western blot. ${ }^{*} p<0.05$; ${ }^{* *} p<0.01{ }^{* * *} p<0.001$ compared to Untreated. 
ER stress exacerbates mitochondrial dysfunction by activating caspase- 9 and increasing the release of cytochrome c [18]. CHOP, DNA damage inducible gene 153 (GADD153), is the main apoptotic factor activated by ER stress, and its overexpression promotes apoptosis in cancer [19]. After treatment with AAEE, AAEE-Pe, and AAEE-Ea for $24 \mathrm{~h}$, the levels of CHOP were detected by Western blot. The results showed that AAEE, AAEE-Pe, and AAEE-Ea significantly increased the levels of CHOP in BEL-7404 and H22 cells (Figure 5C), suggesting that ER stress might be involved in the induction of apoptosis by AAEE, AAEE-Pe, and AAEE-Ea.
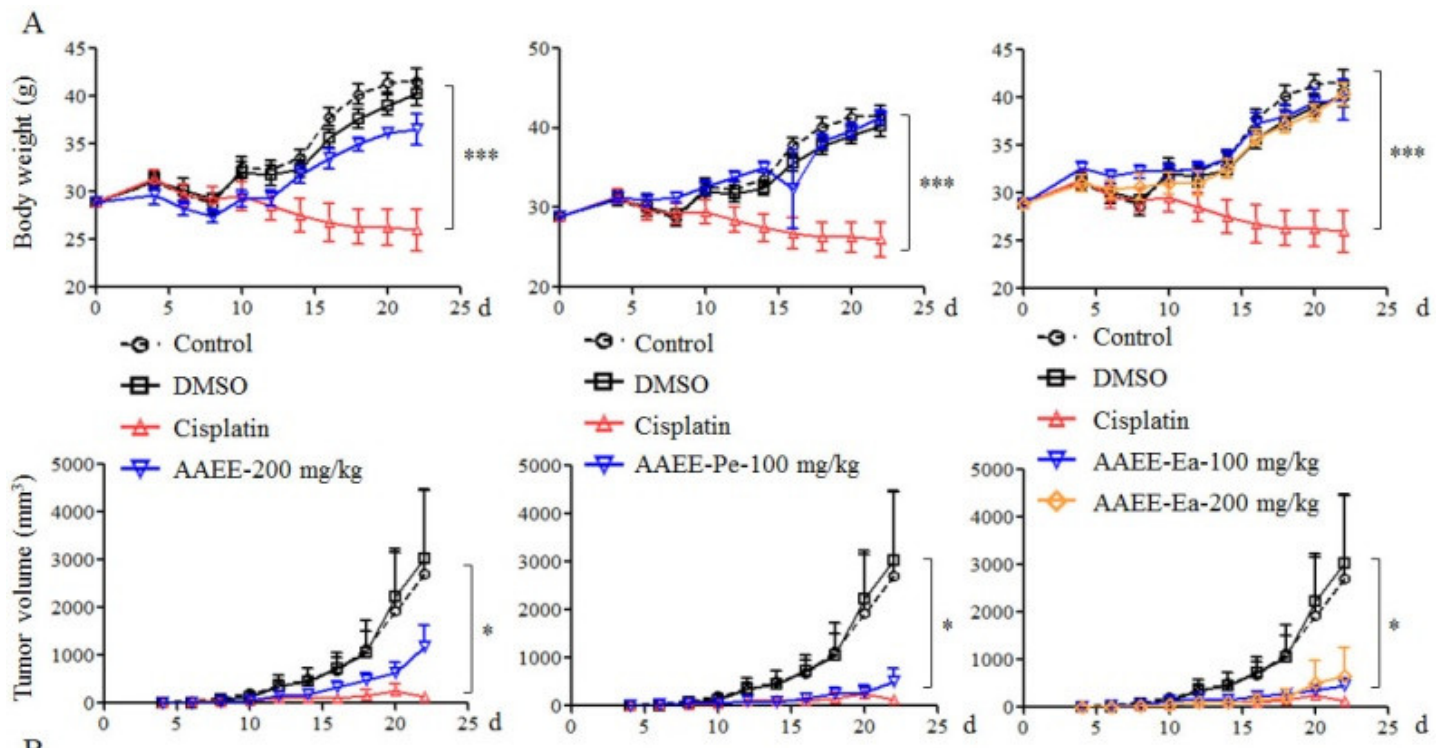

๑ DMSO

\pm Cisplatin

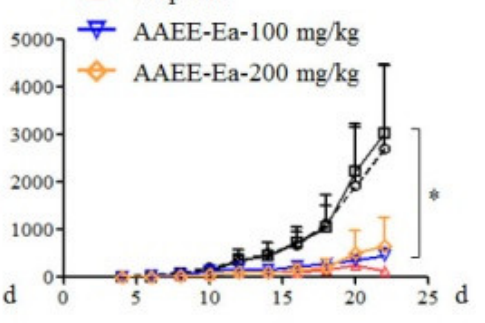

B
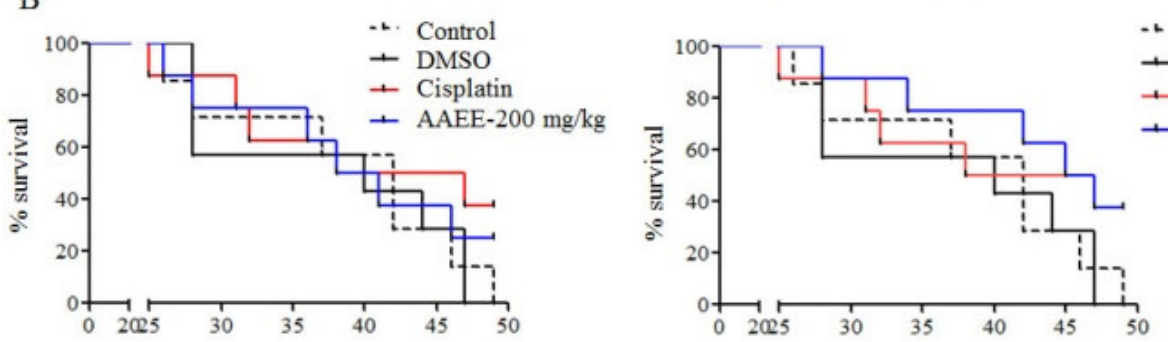

$\therefore$ Control

+ DMSO

+ Cisplatin

+ AAEE-Pe-100 mg/kg

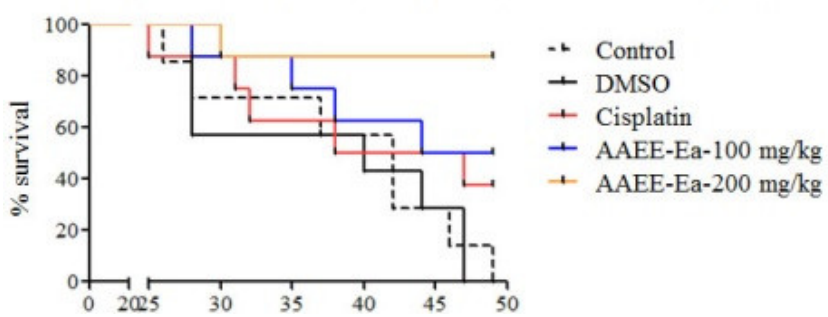

Figure 6. AAEE, AAEE-Pe, and AAEE-Ea suppressed tumor growth in vivo. A tumor mouse model was established by injection of $\mathrm{H} 22$ cells. After 3 days, tumor mice were treated with DMSO, cisplatin, AAEE, AAEE-Pe, and AAEE-Ea. Body weight of mice, tumor sizes (A), and survival rates (B) were monitored at the indicated time points. ${ }^{*} p<0.05 ;{ }^{* * *} p<0.001$ compared to control.

\subsection{AAEE, AAEE-Pe, and AAEE-Ea Inhibit the Growth of H22 Cells In Vivo}

To further confirm the inhibitory effect of AAEE, AAEE-Pe, and AAEE-Ea on tumor growth in vivo, an $\mathrm{H} 22$ tumor mouse model was established in Kunming mice. Tumor mice were treated with different doses of AAEE, AAEE-Pe, and AAEE-Ea after 3 days of H22 cell injection. Cisplatin was used as a positive control and DMSO was used as a solvent control. We observed that cisplatin significantly reduced the weight of mice, but AAEE, AAEE-Pe, and AAEE-Ea did not affect the weight of mice. The tumor growth was significantly inhibited in the cisplatin, $100 \mathrm{mg} / \mathrm{kg}$ AAEE-Pe, and 100 
and $200 \mathrm{mg} / \mathrm{kg}$ AAEE-Ea groups. AAEE at a $200 \mathrm{mg} / \mathrm{kg}$ dose also suppressed tumor growth to a certain degree (Figure 6A). At the end of the tumor study, the survival rates of tumor mice in each group were calculated. All mice were dead in the control (7 out of 7) and DMSO (7 out of 7) groups. The survival rates were $37.5 \%, 25 \%, 37.5 \%, 50 \%$, and $87.5 \%$ in the cisplatin (3 out of 8 ), $200 \mathrm{mg} / \mathrm{kg}$ AAEE (2 out of 8), $100 \mathrm{mg} / \mathrm{kg}$ AAEE-Pe (3 out of 8), and 100 (4 out of 8 ) and $200 \mathrm{mg} / \mathrm{kg}$ (7 out of 8 ) AAEE-Ea groups, respectively (Figure 6B). These data suggest that AAEE, AAEE-Pe, and AAEE-Ea effectively inhibited the growth of $\mathrm{H} 22$ cells in vivo and improved the survival of tumor mice without obvious toxicity.

\section{Discussion}

Chinese herbal medicine (CHM) has a long history of use in treating cancers and provides potential antitumor remedies. A. absinthium, a kind of CHM, has been used as an antipyretic, antiseptic, and anti-parasitic agent for the treatment of chronic fevers and inflammation of the liver [20]. In this study, we prepared AAEE, AAEE-Pe, and AAEE-Ea and investigated their antitumor effects on hepatoma cells. We found that AAEE, AAEE-Pe, and AAEE-Ea significantly suppressed the growth of BEL-7404 and $\mathrm{H} 22$ cells, induced apoptosis and cell cycle arrest, reduced $\Delta \psi \mathrm{m}$, increased the release of cytochrome c, activated caspases, and promoted ROS production and ER stress.

It has been reported that a number of components of CHM can inhibit the growth of tumor cells both in vitro and in vivo, such as polysaccharides, flavones, terpenoids, and phenols [21-26]. The components of polysaccharides, flavones, and triterpenes in AAEE, AAEE-Pe, and AAEE-Ea were quantified. Although the three extracts contained different concentrations of polysaccharides, flavones, and triterpenes, they had similar antitumor effects. The results suggest that polysaccharides, flavones, and triterpenes might not be the major antitumor components in AAEE, AAEE-Pe, and AAEE-Ea. We will further identify the major antitumor components in the extracts of A. absinthium in future study.

The BCL-2 protein family strictly controls the apoptosis of cells [27,28]. An imbalance of proteins in the BCL-2 family triggers the intrinsic apoptosis pathway that increases mitochondrial permeability and the release of cytochrome $c$ and activates caspase-9/caspase-3 [29]. We found that the levels of Bax/Bcl2 significantly increased in BEL-7404 and H22 cells after AAEE, AAEE-Pe, and AAEE-Ea treatment, which might cause the reduction of $\Delta \psi \mathrm{m}$ and the release of cytochrome c to activate caspases and apoptosis. Similarly, Shafi et al. [30] reported that the methanol extract of A. absinthium induced the apoptosis of human breast cancer cells through the modulation of BCL-2 family proteins.

Accumulating evidence points to the role of ER stress in the induction of apoptosis in various cancer cells [31-33]. ER stress exacerbates mitochondrial dysfunction by activating caspase-9 and increasing the release of cytochrome c [34,35]. Our results showed that AAEE, AAEE-Pe, and AAEE-Ea treatment significantly increased the expression of the ER stress-related protein CHOP, indicating that AAEE, AAEE-Pe, and AAEE-Ea might induce apoptosis of BEL-7404 and H22 cells through ER stress and the mitochondria-dependent pathway.

We observed that the ROS levels in hepatoma cells treated with AAEE and AAEE-Pe were lower than that in untreated cells at the beginning of $3 \mathrm{~h}$, although they were significantly upregulated after $6 \mathrm{~h}$, suggesting that the extracts might have antioxidant activities. This is similar to results found in other studies [16,17]. The increased ROS might be due to the reduction of $\Delta \psi \mathrm{m}$ induced by AAEE and AAEE-Pe treatment.

Finally, the antitumor and side effects of AAEE, AAEE-Pe, and AAEE-Ea were evaluated in an H22 tumor mouse model. All three extracts did not affect the weight of mouse but cisplatin significantly reduced the weight of mouse, suggesting that AAEE, AAEE-Pe, and AAEE-Ea might have no side effects in vivo. Moreover, AAEE-Pe and AAEE-Ea significantly inhibited tumor growth, which was similar with cisplatin. AAEE, AAEE-Pe, and AAEE-Ea further improved the survival of H22 tumor mice, and the high dose of AAEE-Ea showed the highest survival rate.

In conclusion, AAEE, AAEE-Pe, and AAEE-Ea inhibited the growth of hepatoma cells through the induction of apoptosis that might be mediated by ER stress and the mitochondria-dependent pathway. 
AAEE, AAEE-Pe, and AAEE-Ea suppressed H22 tumor growth and improved the survival of H22 tumor mice without obvious side effects, indicating that the three extracts might be used to develop safe and effective antitumor agents.

\section{Materials and Methods}

\subsection{Preparation of $A A E E, A A E E-P e$, and $A A E E-E a$}

The AAEE, AAEE-Pe, and AAEE-Ea were prepared according to the following procedure. Briefly, the powder was made using the aerial parts of $A$. absinthium including stems, leaves, flowers, and seeds (Alikang Uygur medicine technology co., Ltd., Urumqi, Xinjiang, China) and extracted overnight using 10 volumes of distilled water at $4{ }^{\circ} \mathrm{C}$. After centrifugation at $8000 \mathrm{rpm}$ for 20 min, the pellet was collected and extracted with 10 volumes of distilled water for $2 \mathrm{~h}$ at $60{ }^{\circ} \mathrm{C}$. After centrifugation, the pellet was extracted using 10 volumes of $85 \%$ ethanol at $60{ }^{\circ} \mathrm{C}$ three times ( $2 \mathrm{~h} /$ time). The supernatant was collected after filtration and concentrated by a rotary evaporator to obtain the extractum. Some extractum was dried by a vacuum freeze-dryer to obtain AAEE. The remaining extractum was dissolved in distilled water and extracted by an equal volume of petroleum ether eight times, then the upper layer was collected and dried by a vacuum freeze-dryer to obtain AAEE-Pe. The bottom layer was extracted by an equal volume of ethyl acetate eight times, then the supernatant was collected and dried by a vacuum freeze-dryer to obtain AAEE-Ea. The AAEE, AAEE-Pe, and AAEE-Ea were dissolved in dimethyl sulfoxide (DMSO) (Sigma, St. Louis, MO, USA) and filtered with a $0.22 \mu \mathrm{m}$ filter. The contents of flavonoids, terpenoids, and polysaccharides were determined by $\mathrm{AlCl}_{3}-\mathrm{KA}_{\mathrm{C}}$, vanillin-glacial acetic acid, and anthrone-sulfuric acid colorimetry, respectively, which were shown in Table 2.

Table 2. The contents of polysaccharides, flavonoids, and triterpenoids in AAEE, AAEE-Pe, and AAEE-Ea.

\begin{tabular}{cccc}
\hline & AAEE & AAEE-Pe & AAEE-Ea \\
\hline Polysaccharides & $31.15 \%$ & $0.68 \%$ & $9.62 \%$ \\
Flavonoids & $16.89 \%$ & $10.26 \%$ & $24.97 \%$ \\
Triterpenes & $22.57 \%$ & $31.59 \%$ & $29.89 \%$ \\
\hline
\end{tabular}

\subsection{Cell Culture}

The NCTC1469, H22, and BEL-7404 cells were obtained from the Xinjiang Key Laboratory of Biological Resources and Genetic Engineering, Xinjiang University (Urumqi, Xinjiang, China) and cultured in Roswell Park Memorial Institute (RPMI) 1640 medium (Gibco, Thermo Fisher Scientific, Waltham, MA, USA) supplemented with 10\% fetal bovine serum (MRC, Changzhou, China) and 1\% L-glutamine (100 mM), $100 \mathrm{U} / \mathrm{mL}$ penicillin, and $100 \mu \mathrm{g} / \mathrm{mL}$ streptomycin (MRC, Changzhou, China) at $37^{\circ} \mathrm{C}$ in humidified air with $5 \% \mathrm{CO}_{2}$.

\subsection{Cell Viability Assay}

The proliferation of NCTC1469, H22, and BEL-7404 cells was analyzed by 3-(4,5-dimethylthiazol-2-yl)2,5-diphenyltetrazolium bromide (MTT) (Sigma, Louis, MO, USA) assay. Briefly, cells (5000 cells/well) were seeded in 96-well plates and treated with various doses of AAEE, AAEE-Pe, and AAEE-Ea for $24 \mathrm{~h}, 48 \mathrm{~h}$, or $72 \mathrm{~h}$. DMSO $(0.3 \%)$ was used as a solvent control and cisplatin $(35 \mu \mathrm{g} / \mathrm{mL})$ was used as a positive control. The supernatant was discarded after centrifugation at $1200 \mathrm{rpm}$ for $5 \mathrm{~min}$ and $100 \mu \mathrm{L}$ of MTT solution $\left(0.5 \mathrm{mg} / \mathrm{mL}\right.$ in PBS) was added to each well and incubated at $37^{\circ} \mathrm{C}$ for $3 \mathrm{~h}$. The formed formazan crystals were dissolved in $200 \mu \mathrm{L}$ DMSO. The $\mathrm{OD}_{490}$ values were measured by a 96-well microplate reader (Bio-Rad Laboratories, Hercules, CA, USA). The relative cell viability was calculated according to the formula Cell viability $(\%)=\left(\mathrm{OD}_{\text {treated }} / \mathrm{OD}_{\text {untreated }}\right) \times 100 \%$. This experiment was conducted three times independently. 


\subsection{Observation of Cell Morphology}

$\mathrm{H} 22$ and BEL-7404 cells were seeded in 96-well plates and were treated with different concentrations of AAEE, AAEE-Pe, and AAEE-Ea for $24 \mathrm{~h}$. After treatment, the morphology of H22 and BEL-7404 cells was observed by inverted fluorescence microscope (Nikon Eclipse Ti-E, Tokyo, Japan).

\subsection{Analysis of Apoptosis}

H22 and BEL-7404 cells were treated with different concentrations of AAEE, AAEE-Pe, and AAEE-Ea for $24 \mathrm{~h}$, and then stained with an Annexin V-FITC/propidium iodide (PI) Apoptosis Detection Kit (YEASEN, Shanghai, China) according to the manufacturer's instructions. Cisplatin and DMSO were used as positive and negative controls, respectively. Samples were analyzed by flow cytometry (BD FACSCalibur, San Jose, CA, USA). This experiment was conducted three times independently.

\subsection{Hoechst 33258 Staining}

H22 and BEL-7404 cells were seeded in 6-well plates at the concentration of $1 \times 10^{5}$ cells/well in $2 \mathrm{~mL}$ medium. After $60 \% \sim 70 \%$ confluence, the cells were treated with AAEE, AAEE-Pe, and AAEE-Ea for $24 \mathrm{~h}$. The cells were collected and fixed with $4 \%$ ice-cold Paraformaldehyde at $4{ }^{\circ} \mathrm{C}$ for $10 \mathrm{~min}$. After washing with PBS, cells were stained with Hoechst 33258 (Beyotime, Shanghai, China) at $4{ }^{\circ} \mathrm{C}$ for $10 \mathrm{~min}$. Samples were observed using an inverted fluorescence microscope.

\subsection{Analysis of the Cell Cycle}

$\mathrm{H} 22$ and BEL-7404 cells were inoculated in $60 \mathrm{~mm}$ culture dishes and treated with different concentrations of AAEE, AAEE-Pe, and AAEE-Ea for $24 \mathrm{~h}$. All cells were collected and washed twice with PBS, then fixed in $70 \%$ ice-cold ethanol overnight at $4{ }^{\circ} \mathrm{C}$. After washing twice with PBS, cells were re-suspended in $250 \mu \mathrm{L}$ propidium iodide/RNase staining buffer (BD Biosciences, San Jose, CA, USA). After $10 \mathrm{~min}$ at room temperature, samples were collected by flow cytometry and the cell cycle distribution was analyzed using ModFit LT 3.0 software (BD FACS Calibur, San Jose, AC, USA). This experiment was conducted three times independently.

\subsection{Analysis of $\Delta \psi m$}

H22 and BEL-7404 cells were treated with different concentrations of AAEE, AAEE-Pe, and AAEE-Ea for $24 \mathrm{~h}$ and then stained with the membrane-permeable JC-1 dye (Beyotime, Shanghai, China) for $30 \mathrm{~min}$ at $37^{\circ} \mathrm{C}$. Samples were analyzed by flow cytometry. This experiment was conducted three times independently.

\subsection{Analysis of ROS}

BEL-7404 cells were treated with AAEE, AAEE-Pe, and AAEE-Ea for 2, 4, 6, 12, and $24 \mathrm{~h}$. H22 cells were treated with AAEE, AAEE-Pe, and AAEE-Ea for $24 \mathrm{~h}$. Cells were stained by $10 \mathrm{mM}$ of fluorescent probe $2^{\prime}, 7^{\prime}$-dichlorodihydrofluorescein diacetate (DCFH-DA) (Beyotime, Shanghai, China) for $20 \mathrm{~min}$ at $37^{\circ} \mathrm{C}$. After washing three times with ice-cold PBS, samples were analyzed by flow cytometry. This experiment was conducted two times independently.

\subsection{Western Blot}

The antibodies against caspase-9, Bax and Bcl-2, and anti-mouse IgG-HRP and anti-rabbit IgG-HRP were purchased from BBI Life Sciences (Shanghai, China). The antibodies against caspase-3, PARP, cytochrome $c$, and $\beta$-actin were obtained from Cell Signaling Technology (Danvers, MA, USA). The antibodies against $\mathrm{CHOP}$ and CyclinB1 were bought from Beyotime (Shanghai, China).

H22 and BEL-7404 cells were treated with AAEE, AAEE-Pe, and AAEE-Ea for $24 \mathrm{~h}$. After washing twice with PBS, cell lysates were prepared with RIPA Lysis Buffer (Beijing ComWin Biotech Co., 
Ltd., Beijing, China) and protein concentrations were detected using a BCA Kit (Thermo Fisher Scientific, Waltham, MA, USA) according to the manufacturer's instructions. Proteins were separated on $12 \%$ sodium dodecyl sulfate polyacrylamide gel electrophoresis (SDS-PAGE) and transferred to a polyvinylidene difluoride (PVDF) membrane. After incubation with primary and secondary antibodies, target proteins were detected by chemiluminescence (Beyotime, Shaghai, China). Signals were quantified using ImageJ digitizing software (ImageJ 1.50, National Institutes of Health, Bethesda, MD, USA). This experiment was conducted three times independently.

\subsection{Animals and Ethics Statement}

Six- to eight-week-old male Kunming mice were purchased from Animal Laboratory Center, Xinjiang Medical University (Urumqi, Xinjiang, China). Mice were kept in a standard temperature-controlled, light-cycled animal facility at Xinjiang University. All animal experiments were approved by the Committee on the Ethics of Animal Experiments of Xinjiang Key Laboratory of Biological Resources and Genetic Engineering (BRGE-AE001) and performed under the guidelines of the Animal Care and Use Committee of College of Life Science and Technology, Xinjiang University.

\subsection{Tumor Mouse Study}

For establishment of a tumor mouse model, male Kunming mice were injected with $1 \times 10^{6} \mathrm{H} 22$ cells in $100 \mu \mathrm{L}$ PBS subcutaneously. After 3 days, mice were randomly divided into seven groups (7 mice/group for Control and DMSO, 8 mice/group for the other five groups). The solvent control group intraperitoneally received $0.1 \mathrm{~mL}$ DMSO daily. The positive group was intraperitoneally injected with $5 \mathrm{mg} / \mathrm{kg}$ cisplatin at intervals of five days. The experimental groups were intraperitoneally injected with $200 \mathrm{mg} / \mathrm{kg}$ AAEE, $100 \mathrm{mg} / \mathrm{kg}$ AAEE-Pe, or $100 \mathrm{mg} / \mathrm{kg}$ or $200 \mathrm{mg} / \mathrm{kg}$ AAEE-Ea in $0.1 \mathrm{~mL}$ DMSO every two days. Tumor sizes were measured using calipers and tumor volume was calculated according to the formula tumor volume $\left(\mathrm{mm}^{3}\right)=\left(\right.$ length $\times$ width $\left.{ }^{2}\right) / 2$.

\subsection{Statistical Analysis}

The data are expressed as mean \pm standard error of the mean (SEM). Statistical significance was analyzed using one-way analysis of variance (ANOVA) by Tukey's Multiple Comparison Test. $p<0.05$ was considered statistically significant.

Author Contributions: X.W., D.Z., Q.C., R.L. and X.X. performed the experiments; L.X. and J.L. designed the experiments, analyzed the data, and wrote the paper.

Funding: This work was supported by the Chinese National Natural Science Foundation Grant [U1803381 to Jinyao Li and 31860258 to Lijie Xia], the 1000 Young Talents Program of China to Jinyao Li and the "Tianshan Youth Project" Young Ph.D. Science and Technology talents Project [no. 2017Q077] to Lijie Xia.

Conflicts of Interest: The authors declare no conflict of interest.

\section{References}

1. Wang, D.; Sun, Q.; Wu, J.; Wang, W.; Yao, G.; Li, T.; Li, X.; Li, L.; Zhang, Y.; Cui, W.; et al. A new Prenylated Flavonoid induces G0/G1 arrest and apoptosis through p38/JNK MAPKpathways in Human Hepatocellular Carcinoma cells. Sci. Rep. 2017, 7, 5736. [CrossRef] [PubMed]

2. Bray, F.; Ferlay, J.; Soerjomataram, I.; Siegel, R.L.; Torre, L.A.; Jemal, A. Global cancer statistics 2018: GLOBOCAN estimates of incidence and mortality worldwide for 36 cancers in 185 countries. CA-A Cancer J. Clin. 2018, 68, 394-424. [CrossRef] [PubMed]

3. London, W.T.; Petrick, J.L.; McGlynn, K.A.; Thun, M.J.; Linet, M.S.; Cerhan, J.R.; Haiman, C.A.; Schottenfeld, D. (Eds.) Cancer Epidemiology and Prevention, 4th ed.; Oxford University Press: New York, NY, USA, 2018; pp. 635-660.

4. Mauer, K.; O'Kelley, R.; Podda, N.; Flanagan, S.; Gadani, S. New treatment modalities for hepatocellular cancer. Curr. Gastroenterol. Rep. 2015, 17, 19. [CrossRef] [PubMed] 
5. Wang, Y.; Deng, T.; Zeng, L.; Chen, W. Efficacy and safety of radiofrequency ablation and transcatheter arterial chemoembolization for treatment of hepatocellular carcinoma: A meta-analysis. Hepatology 2016, 46, 58-71. [CrossRef] [PubMed]

6. Liu, G.; Fan, X.; Tang, M.; Chen, R.; Wang, H.; Jia, R.; Zhou, X.; Jing, W.; Wang, H.J.; Yang, Y.; et al. Osteopontin induces autophagy to promote chemo-resistance in human hepatocellular carcinoma cells. Cancer Lett. 2016, 383, 171-182. [CrossRef] [PubMed]

7. Horgan, A.M.; Dawson, L.A.; Swaminath, A.; Knox, J.J. Sorafenib and radiation therapy for the treatment of advanced hepatocellular carcinoma. J. Gastrointest. Cancer 2012, 43, 344-348. [CrossRef] [PubMed]

8. Tabernero, J.; Garcia-Carbonero, R.; Cassidy, J.; Sobrero, A.; Van, C.E.; Kohne, C.H.; Tejpar, S.; Gladkov, O.; Davidenko, I.; Salazar, R.; et al. Sorafenib in combination with oxaliplatin, leucovorin, and fluorouracil (modified FOLFOX6) as first-line treatment of metastatic colorectal cancer: The respect trial. Clin. Cancer Res. 2013, 19, 2541-2550. [CrossRef] [PubMed]

9. Kamel, M.; Nidhal, S.; Olfa, B.; Slim, D.; Sonia, T.; Abdulkhaleg, A.; Khaldoun, A.S.; Wided, B.A.; Sana, A.; Adel, H.B.; et al. Chemical composition and antioxidant and antimicrobial activities of wormwood (Artemisia absinthium L.) essential oils and phenolics. J. Chem. 2015, 2015, 1-12.

10. Azizi, K.; Shahidi-Hakak, F.; Asgari, Q.; Hatam, G.R.; Fakoorziba, M.R.; Miri, R.; Moemenbellah-Fard, M.D. In vitro efficacy of ethanolic extract of Artemisia absinthium (Asteraceae) against Leishmania major L. using cell sensitivity and flow cytometry assays. J. Parasit. Dis. 2016, 40, 735-740. [CrossRef] [PubMed]

11. Tamargo, B.; Monzote, L.; Piñón, A.; Machín, L.; García, M.; Scull, R.; Setzer, W.N. In vitro and in vivo evaluation of essential oil from Artemisia absinthium L. formulated in nanocochleates against cutaneous Leishmaniasis. Medicines 2017, 4, 38. [CrossRef] [PubMed]

12. Turak, A.; Shi, S.P.; Jiang, Y.; Tu, P.F. Dimeric guaianolides from Artemisia absinthium. Phytochemistry 2014, 105, 109-114. [CrossRef] [PubMed]

13. Caner, A.; Doskaya, M.; Degirmenci, A.; Can, H.; Baykan, S.; Uner, A.; Basdemir, G.; Zeybek, U.; Guruz, Y. Comparison of the effects of Artemisia vulgaris and Artemisia absinthium growing in western Anatolia against trichinellosis (Trichinella spiralis) in rats. Exp. Parasitol. 2008, 119, 173-179. [CrossRef] [PubMed]

14. Amat, N.; Upur, H.; Blazeković, B. In vivo hepatoprotective activity of the aqueous extract of Artemisia absinthium L. againstchemically and immunologically induced liver injuries in mice. J. Ethnopharmacol. 2010, 131, 478-484. [CrossRef] [PubMed]

15. Gilani, A.H.; Janbaz, K.H. Preventive and curative effects of Artemisia absinthium on acetaminophen and CCl4-induced hepatotoxicity. Gen. Pharmacol. 1995, 26, 309. [CrossRef]

16. Craciunescu, O.; Constantin, D.; Gaspar, A.; Toma, L.; Utoiu, E.; Moldovan, L. Evaluation of antioxidant and cytoprotective activities of Arnica montana L. and Artemisia absinthium L. ethanolic extracts. Chem. Cent. J. 2012, 6, 1-11. [CrossRef] [PubMed]

17. Bora, K.S.; Sharma, A. Evaluation of antioxidant and free-radical scavenging potential of Artemisia absinthium. Pharm. Biol. 2011, 49, 1216. [CrossRef] [PubMed]

18. Zhang, M.Z.; Du, H.X.; Huang, Z.X.; Zhang, P.; Yue, Y.Y.; Wang, W.Y.; Liu, W.; Zeng, J.; Ma, J.B.; Chen, G.Q.; et al. Thymoquinone induces apoptosis in bladder cancer cell via endoplasmic reticulum stress-dependent mitochondrial pathway. Chem.-Biol. Interact. 2018, 292, 65-75. [CrossRef]

19. Vuyolwethu, S.; Georgia, S.; Roger, H.; Grafov, A.; Grafova, I.; Nieger, M.; Katz, A.A.; Parker, M.I.; Kaschula, C.H. The cytotoxicity of the ajoene analogue BisPMB in WHCO1 oesophageal cancer cells is mediated by CHOP/GADD153. Molecules 2017, 22, 892. [CrossRef]

20. Zhang, H.P. Drug Standard of Ministry of Public Health of the People's Republic of CHINA Xingjiang Technological and Health Publishing House, Xingjiang, Uighur Medicine Part; Xinjiang Publishing House of Science: Urumqi, China, 1999; p. 53.

21. Song, G.C.; Yu, Y.J.; Wang, X.J. Experiments on antitumor activity of Scutellaria barbata polysaccharides and its immunological mechanisms. Open Access Libr. J. 2011, 13, 641-643.

22. Wang, D.D.; Wu, Q.X.; Pan, W.J.; Hussain, S.; Mehmood, S.; Chen, Y. A novel polysaccharide from the Sarcodon aspratus triggers apoptosis in Hela cells via induction of mitochondrial dysfunction. Food Nutr. Res. 2018, 62, 1285. [CrossRef]

23. Ashokkumar, R.; Jamuna, S.; Sakeena Sadullah, M.S.; Niranjali, D.S. Vitexin protects isoproterenol induced post myocardial injury by modulating hipposignaling and ER stress responses. Biochem. Biophys. Res. Commun. 2018, 496, 731-737. [CrossRef] [PubMed] 
24. Fan, C.; Yang, Y.; Liu, Y.; Jiang, S.; Di, S.Y.; Hu, W.; Ma, Z.Q.; Li, T.; Zhu, Y.F.; Xin, Z.L.; et al. Icariin displays anticancer activity against human esophageal cancer cells via regulating endoplasmic reticulum stress-mediated apoptotic signaling. Sci. Rep. 2016, 6, 21145. [CrossRef] [PubMed]

25. Cai, Y.L.; Zheng, Y.F.; Gu, J.Y.; Wang, S.Q.; Wang, N.; Yang, B.W.; Zhang, F.X.; Wang, D.M.; Fu, W.J.; Wang, Z.Y. Betulinic acid chemosensitizes breast cancer by triggering ER stress-mediated apoptosis by directly targeting GRP78. Cell Death Dis. 2018, 9, 636. [CrossRef] [PubMed]

26. Wang, B.; Zhou, T.Y.; Nie, C.H.; Wang, D.L.; Zheng, S.S. Bigelovin, a sesquiterpene lactone, suppresses tumor growth through inducing apoptosis and autophagy via the inhibition of mTOR pathway regulated by ROS generation in liver cancer. Biochem. Biophys. Res. Commun. 2018, 499, 156-163. [CrossRef] [PubMed]

27. Ramos, S. Effects of dietary flavonoids on apoptotic pathways related to cancer chemoprevention. J. Nutr. Biochem. 2007, 18, 427-442. [CrossRef]

28. Marzo, I.; Naval, J. Bcl-2 family members as molecular targets in cancer therapy. Biochem. Pharmacol. 2008, 76, 939-946. [CrossRef]

29. Garner, T.P.; Lopez, A.; Reyna, D.E.; Spitz, A.Z.; Gavathiotis, E. Progress in targeting the BCL-2 family of proteins. Curr. Opin. Chem. Biol. 2017, 39, 133-142. [CrossRef]

30. Shafi, G.; Hasan, T.N.; Syed, N.A.; Al-Hazzani, A.A.; Alshatwi, A.A.; Jyothi, A.; Munshi, A. Artemisia absinthium( $A A)$ : A novel potential complementary and alternative medicine for breast cancer. Mol. Biol. Rep. 2012, 39, 7373-7379. [CrossRef]

31. Banerjee, A.; Banerjee, V.; Czinn, S.; Blanchard, T. Increased reactive oxygen species levels cause ER stress and cytotoxicity in andrographolide treated colon cancer cells. Oncotarget 2017, 8, 26142-26153. [CrossRef]

32. Yu, X.S.; Du, J.; Fan, Y.J.; Liu, F.J.; Cao, L.L.; Liang, N.; Xu, D.G.; Zhang, J.D. Activation of endoplasmic reticulum stress promotes autophagy and apoptosis and reverses chemoresistance of human small cell lung cancer cells by inhibiting the PI3K/AKT/mTOR signaling pathway. Oncotarget 2016, 7, 76827-76839. [CrossRef]

33. Merlot, A.; Shafie, N.; Yu, Y.; Richardson, V.; Jansson, P.J.; Sahni, S.; Lane, D.J.; Kovacevic, D.S.; Richardson, D.R. Mechanism of the induction of endoplasmic reticulum stress by the anti-cancer agent, di-2-pyridylketone 4,4-dimethyl-3-thiosemicarbazone (Dp44mT): Activation of PERK/eIF2a, IRE1a, ATF6 and calmodulin kinase. Biochem. Pharmacol. 2016, 109, 27-47. [CrossRef] [PubMed]

34. Wu, M.H.; Chiou, H.L.; Lin, C.L.; Lin, C.Y.; Yang, S.F.; Hsieh, Y.H. Induction of endoplasmic reticulum stress and mitochondrial dysfunction dependent apoptosis signaling pathway in human renal cancer cells by Norcantharidin. Oncotarget 2018, 9, 4787-4797. [CrossRef] [PubMed]

35. Zhang, D.; Gao, C.; Li, R.; Zhang, L.; Tian, J.K. TEOA, a triterpenoid from Actinidia eriantha, induces autophagy in SW620 cells via endoplasmic reticulum stress and ROS-dependent mitophagy. Arch. Pharm. Res. 2017, 40, 1-13. [CrossRef] [PubMed]

Sample Availability: Samples of the compounds are available from the authors.

(C) 2019 by the authors. Licensee MDPI, Basel, Switzerland. This article is an open access article distributed under the terms and conditions of the Creative Commons Attribution (CC BY) license (http:/ / creativecommons.org/licenses/by/4.0/). 